\title{
Disease Insights Through Analysis: Using machine learning to provide feedback in the MONARCA system
}

\author{
Mads Frost, Jakob E. Bardram \\ Pervasive Interaction Technology Lab \\ IT University of Copenhagen \\ Copenhagen, Denmark \\ Email: \{madsf, bardram $\} @$ itu.dk
}

\author{
Afsaneh Doryab \\ Human-Computer Interaction Institute \\ Carnegie Mellon University \\ Pittsburgh, PA, USA \\ Email: adoryab@cs.cmu.edu
}

\begin{abstract}
There is currently a growing interest in personal health technologies using data collection strategies to develop context-aware systems. The insights from this data could help patients and clinicians monitor and manage mental illness. We describe our approach to support the data analysis and feedback to clinicians and patients through extending the MONARCA SelfAssessment System.
\end{abstract}

\section{INTRODUCTION}

There is a growing interest in personal monitoring systems that use different data collection approaches to develop context-aware systems. These systems can help users by enabling them to monitor and visualize their behaviors, reminding them to perform specific tasks, and providing feedback on the effectiveness of their behaviors.

Personal monitoring systems have been suggested for the management of a wide range of health-related conditions, and comprehensive electronic monitoring systems have been presented especially for patients suffering from bipolar disorder [1], [2], [3]. Bipolar disorder is associated with a high risk of relapse and hospitalization [4]. Major reasons for the decreased effect of interventions in clinical practice are delayed intervention for depressive and manic episodes [5], [6] and limitations of paper-based mood charts [7].

However, these monitoring systems collect a vast amount of data that contain a lot of information which can be difficult for the patients and clinicians to make sense of. Due to the complexity of the illness, it is unclear what data are most important as symptoms vary from patient to patient, and may be difficult to recognize.

Hence, in this demo, we present the extension of the MONARCA Self-Assessment System [3], which aim at helping patients and clinicians get an insight into important aspects of the patients' mental status using machine learning methods.

\section{SYSTEM DESCRIPTION}

This addition to the MONARCA system collects the subjective and objective data collected through the MONARCA Android application. It runs it through a set of different algorithms on our server, and outputs past and current impact factors as well as a forecast of the patients' mood.

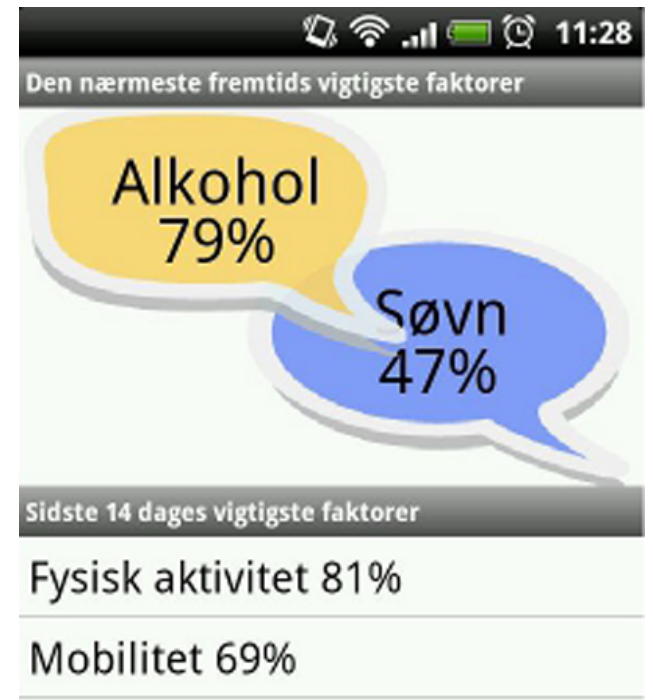

Alkohol 63\%

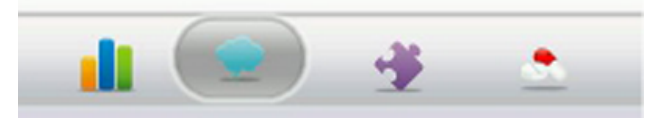

Fig. 1. An example of the impact factor screen from the MONARCA Android application, showing alcohol and sleep as important future impact factors, and physical activity, mobility and alcohol as past impact factors.

The impact factors are presented to the patients in form of a screen in the MONARCA Android application (Fig. 1), which also provides strategies on how to handle the different impact factors. Furthermore, there is an abstract data visualization metaphor created as a Live Wallpaper (Fig. 2), running on the phone, to increase the patients awareness to the information provided by the system.

Past and present impact factors as well as the forecast of the patients mood are presented to clinicians through the web portal. The quick patient overview (Fig. 3) enables them to 


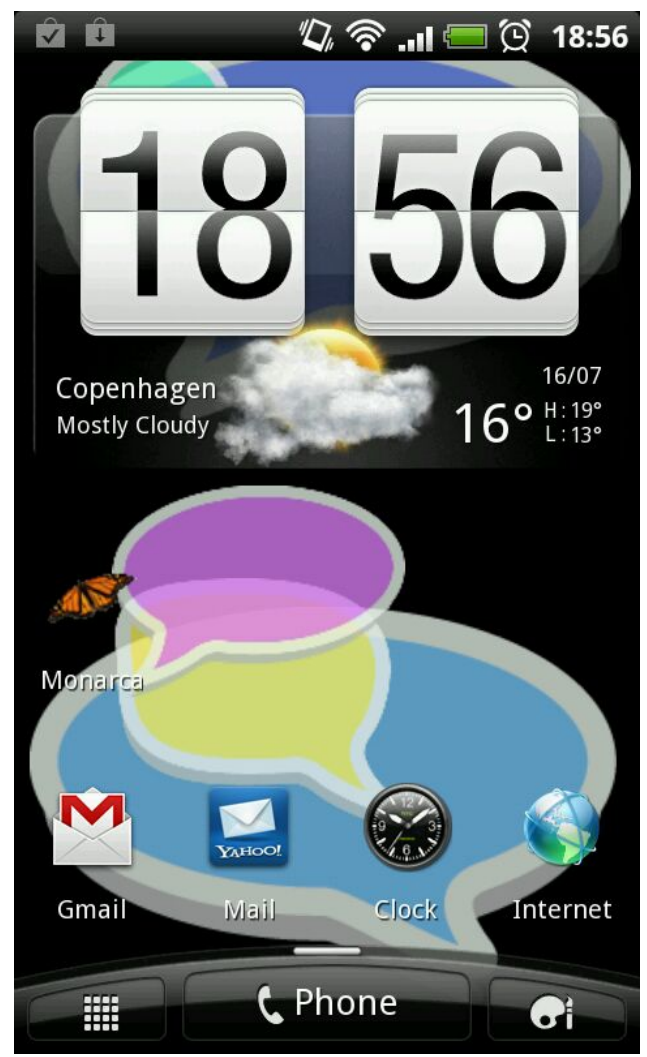

Fig. 2. The MONARCA Live Wallpaper bubble visualization.

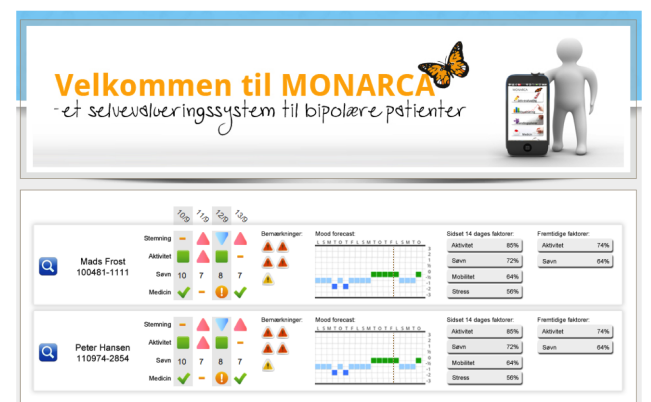

Fig. 3. The MONARCA patient overview screen with mood forecasts and impact factors.

focus on patients in need of immediate attention. The detailed information along with the strategies the patients have been given are available through sub pages of the portal.

At the demo, we will present the technical aspects of the system which include the server side, the algorithms, the Android application, and the web portal. We also provide an insight into how this application is integrated into users daily life. We demonstrate the functionalities that help both patients and clinicians and provide an insight into how this application is integrated into patients daily life.

\section{SCENARIO AND PROJECT VIDEOS}

An overview of the project is provided in the MONARCA introduction movie, which is available in the IT University of Copenhagen's video archive at http://video.itu.dk/video/4550686/monarca
A scenario have been documented in the MONARCA movie available on YouTube at

http://www.youtube.com/watch? $v=U V S O c A x l Q x M$

Note that the background sensing and activity inferring is not part of this scenario.

\section{ACKNOWLEDGMENT}

This work has been done in close collaboration with a group of patients and clinicians from the Copenhagen Affective Disorder Clinic at the University Hospital of Copenhagen. MONARCA is funded as a STREP project under the FP7 European Framework program. More information can be found at http://pit.itu.dk/monarca and http://monarca-project.eu/

\section{REFERENCES}

[1] P. Prociow and J. Crowe, "Towards personalised ambient monitoring of mental health via mobile technologies." Technol Health Care, vol. 18, no. 4-5, pp. 275-284, 2010.

[2] R. Paradiso, A. Bianchi, K. Lau, and E. Scilingo, "Psyche: personalised monitoring systems for care in mental health." Conf Proc IEEE Eng Med Biol Soc, vol. 2010, pp. 3602-3605, 2010.

[3] J. E. Bardram, M. Frost, K. Szántó, and G. Marcu, "The monarca selfassessment system: a persuasive personal monitoring system for bipolar patients," in Proceedings of the 2nd ACM SIGHIT International Health Informatics Symposium, ser. IHI '12. New York, NY, USA: ACM, 2012, pp. 21-30.

[4] P. M. LV Kessing, PK Andersen and T. Bolwig, "Recurrence in affective disorder. i. case register study," British Journal of Psychiatry, vol. 172, pp. 23-28, 2008.

[5] A. J. P. W. C. B. RK Morriss, MA Faizal and J. McCarthy, "Interventions for helping people recognise early signs of recurrence in bipolar disorder," Cochrane Database Syst Rev., vol. Jan 24;(1), no. CD004854, 2007.

[6] K. H. C. G. S. D. A. A. B. F. F. K. M Berk, GS Malhi, "Early intervention in bipolar disorders: clinical, biochemical and neuroimaging imperatives," Journal of Affective Disorders, vol. 114(1-3), pp. 1-13, 2009.

[7] M. Matthews and G. Doherty, "In the mood: engaging teenagers in psychotherapy using mobile phones," in In Proc. ACM CHI 2011, ser. CHI '11. New York, NY, USA: ACM, 2011, pp. 2947-2956. 